\title{
Babylon Berlin, een 'must' voor de culturele criminologie
}

Marc Cools

\section{Berlijn voor criminologen}

We kunnen veronderstellen dat elke criminoloog al wel eens Berlijn heeft bezocht. Wie deze Faust's Metropolis (Richie, 1999) nog niet grondig heeft doorploegd, koestert misschien wel een spreekwoordelijk gat in de eigen intellectuele bagage. Zouden er bovendien ook criminologen bestaan die de boeken Afscheid van Berlijn (1971) van Christopher Isherwood (1904-1986) en Berlijn Alexanderplatz (1989) van Alfred Döblin (1878-1957) nog niet hebben gelezen? Laat ons hopen dat de films Cabaret (1972), Christiane F. - Wir Kinder vom Bahnhof Zoo (1981) en/of de televisiereeks Berlin Alexanderplatz (1980) hun iets zegt. Of zouden er ook collega's zijn die niet weten dat de David Bowie (1947-2016) trilogie: Low (1977), Heroes (1977) en Lodger (1979) en The Idiot (1977) van Iggy Pop Berlijnse roots (Baqué, 2013) hebben? De bovengenoemde kunst (Siegel, 2009), als een van de meest creatieve menselijke uitingsvormen, kunnen de (cultureel) criminoloog immers inspireren en gidsen in zijn voortdurende zoektocht in de tuin van Akademos (Cools, 2018). Berlijn is niet veraf en al dan niet klimaatbewuste city trips zijn nog steeds niet verboden. Op reis dan maar, in de wetenschap dat we kennis en inzichten kunnen verwerven inzake macht, structuur, fenomenen, betekenis en symboliek (Cools, 2019) en dat we deze kunnen plaatsen binnen de cultures of deviance en de cultures of control (Ferrel e.a., 2008).

Onze eerste bezoeken aan Berlijn dateren alweer van de jaren 1980. We koesteren nog steeds de veelvuldig door het Wachregiment Feliks Dzierzynski aangebrachte stempels in onze reispas. De grensovergangen Helmstedt-Marienborg en Checkpoint Charlie doorkruisen was destijds een avontuur op zich. Het wordt misschien ook eens tijd om het eigen dossier te gaan inkijken in het Gauck-Behörde of het Information and Documentation Center of the Office in Charge of Stasi Documents (Cools, 2009: 31). Uitgaan in Ostberlin Hauptstadt der Deutsche Demokratische Republik, onder het toezicht van de in blauw uniform gestoken Freie Deutsche Jugend, moet zeker sporen achtergelaten hebben. Westerse, op de zwarte markt geldwisselende new wavers die verbroederden en verzusterden met DDR-leeftijdsgenoten die bovendien gek waren op de muziek van Depeche Mode en Duran Duran, waren meer dan verdacht en een dossiertje waard. U leest het al, hier schrijft een Berlijnfanaat. Na een tiental bezoeken staat Berlijn vaak opnieuw op het reisprogramma. Er zijn altijd nieuwe criminologische ontdekkingen te doen. 
Men kan een criminologische reis naar Berlijn voorbereiden. Het is immers de stad van de (on)vrijheid en de (in)tolerantie die is doordesemd van haar eigen woelige geschiedenis. Het imperialistische keizerlijke (1871-1918), het nationaalsocialistische (1933-1945) en het communistische (1949-1990) Berlijn versus het Berlijn van de Weimarrepubliek (1918-1933) en de westerse enclave (1961-1989).

Zo kan een criminoloog het imperialistisch keizerlijk Berlijn vatten door bijvoorbeeld de werkzaamheden van de Internationale Kriminalistische Vereinigung (Fijnaut, 2014; Wetzel, 2000) te bestuderen. Naast de dominante conservatieve stroming bestond er toch ook een progressief humanistische visie op de criminologie (Salle, 2014). Het in 1911 opgerichte Kaiser-Wilhelm-Gesellschaft is eveneens historisch relevant. Deze voorloper van het Max-Planck-Gesellschaft met haar talrijke instituten lag mede aan de bron van de zich later ontwikkelende nationaalsocialistische criminologie (Heim e.a., 2009). Hetzelfde gaat op voor de introductie van de vaak raciale eugenetica in de wetenschap (Watson, 2010). De ideologische inbedding van deze criminologie (Saft, 2008) en het gebruik (Rebbein, 1987), maar vooral het misbruik, ervan door de nationaalsocialistische strafrechtsactoren (Ostendorf \& Danker, 2003; Hirsch e.a., 1997) geven meer dan koude rillingen en afschuw. De criminologie beleefde toen haar darkest hour (Rafter, 2008). Ook de communistische (Fijnaut, 2014) dan wel Sozialistische Kriminologie (Buchholz e.a., 1971) spoort naar onze mening ook niet echt anders (Wolfe, 1992; Meador, 1986). Dit werk, samen te lezen met het boek Kriminologie (Lekschas e.a., 1983) geeft een inkijk in de criminologiebeoefening in de DDR (Rode, 1996; Mulder, 1980). Beide standaardwerken waren in de jaren 1980 prominent aanwezig in de Karl Marx-Buchhandlung (Cools, 2009: 29) op de Unter den Linden. Vandaag is de boekhandel er verdwenen en leeft hij vooral verder in de slotscène van de film Das Leben der Anderen (2006). Maar laten we het nu vooral over die toch wel zeer bijzondere, vrije en tolerante Weimarrepubliek hebben.

\section{Babylon Berlin en de kunsttentoonstelling Berlijn 1912-1932}

We zijn de mening toegedaan dat vooral de Weimarrepubliek, als het eerste democratische 'experiment' in Duitsland, de culturele criminoloog moet kunnen boeien. Deze politieke, economische, sociale, seksuele en culturele tot de vrijheid en de tolerantie sprekende episode werd opnieuw de actualiteit in gekatapulteerd door de op de Duitse ARD lopende televisiereeks Babylon Berlin (2017-2018). Babylon lag niet langer aan de Eufraat maar in de Bettler-Metropole (Stürickow, 2019) aan de Spree. Op hetzelfde ogenblik (2018-2019) liep te Brussel in het Koninklijk Museum voor Schone Kunsten de kunsttentoonstelling Berlijn 1912-1932. De daar te koop aangeboden catalogus The New Berlin 1912 - 1932 (Rossi-Schrimpf, 2018) en het opmerkelijke en zeer verzorgde stripverhaal Night Falls on the Berlin of the Roaring Twenties (Nippoldt \& Pofalla, 2018) waren er onmisbare gidsen.

De Weimarrepubliek in het algemeen en Berlijn in het bijzonder werden gekarakteriseerd door een uitzonderlijke bloei en een gevoel van bevrijding uit de autori- 
taire dwang die op ieder niveau aanwezig was. Het militarisme moest plaatsmaken voor een democratisch bestel van de laatste kans. Maar oude gewoontes bleven er een taai leven leiden (De Jonge, 1980). De alom tegenwoordige en dominante geldontwaarding (MacGregor, 2015) en de werkloosheid (Gall, 1984) schiepen echter een antidemocratisch klimaat waarin conservatieven, liberalen, socialisten, nationaalsocialisten en communisten altijd en overal op de straat vochten om de macht op de Staat. Tegen deze achtergrond kunnen criminologen hun gading vinden in de Schmutz und Schund (Weitz, 2007) die Berlijn kenmerkte.

Berlijn was naast de sekshoofdstad van Europa ook een plek waar de criminaliteit welig tierde. Er was een gecontroleerde vrouwelijke en mannelijke prostitutie, maar ook de verboden kinderprostitutie bestond. Het nachtleven kende cabaretten, erotische revues en een open cultuur rond homoseksualiteit. Ook waren er talrijke seksclubs voor lesbische vrouwen en voor de zogenoemde Grenzgänger. Deze BDSM-cultuur vond er vaak een link naar de aanwezige fascinatie voor het occulte en het satanisme. Samengevat was seks het Geschäft der Stadt (Gordon, 2011). Inzake criminaliteit werd Berlijn ook en vooral de hoofdstad van moord, georganiseerde misdaad en politiek geweld (Boegel, 2018; Liang, 1970).

De televisiereeks Babylon Berlin, bestaande uit zestien afleveringen speelt zich af in het samenzweerderige Berlijn van 1929 en is gebaseerd op het werk van Volker Kutscher. Deze in 1962 te Lindlar geboren thrillerschrijver studeerde Germaanse filologie, filosofie en geschiedenis aan de universiteiten van Wuppertal en Köln. Zijn in 2008 verschenen historische Krimi of Kriminalroman met als titel Der nasse Fisch deed Gereon Rath geboren worden. Rath is een opmerkelijke hoofdfiguur in deze atypische whodunit (Wünnenberg, 2014). Het is een apolitieke, of Nichtwähler (Feierabend \& Kaufmann, 2011), en ambitieuze uit Keulen afkomstige inspecteur bij de Berlijnse Mordkommission van de Kriminalpolizei. Deze in de Rode Burcht, zoals het hoofdgebouw op de Alexanderplatz werd genoemd, kantoor houdende politeman domineert de verhalen. Als een Gutmensch wordt hij getraumatiseerd door zijn oorlogservaringen (Kilb \& Körte, 2017) gedreven door rechtvaardigheid in een zich van hem steeds meer vervreemdend Duitsland.

Deze Krimi werd in 2011 ook bekroond met de Reinickendorfer Krimifuchs. Onder regie van Tom Tykwer, Achim von Borries en Henk Handloegten is Babylon Berlin in Duitsland een hype geworden en is het uitkijken naar de derde en/of vierde reeks. Bingewatchers zijn bij dezen gewaarschuwd. Ondersteund door een soundtrack met een passende Klankästhetik (Töteberg, 2018) en dito kostuums, locaties, voertuigen en artefacten verenigt Babylon Berlin seks, criminaliteit en geschiedenis (Jysch, 2018; Ströbele, 2017) in zich. Het Party-Berlin staat er tegenover de Barbarisierung (Kilb \& Körte, 2017). Naast prostitutie en de productie van pornofilms is er ook oog voor moord, zelfmoord, seriemoord, politieke en politionele corruptie, afpersing, oplichting, wapenhandel, drughandel en -gebruik. De georganiseerde misdaad, of de Ringvereine en Ringbrüder (Liang, 1970), met namen zoals Deutsche Kraft, Nordpiraten en Berolina ageert er tussen de straatgevechten van de communisten met de nationaalsocialisten (Diehl, 1977). Het is er een spreekwoordelijk dansen op de vulkaan (Nippoldt \& Pofalla, 2018). In dit Ich bin Babel, 
die Sünderin (Boegel, 2018) wordt alles overgoten en opgesnoven met alcohol en cocaine in het uitgebreid aanwezige en gevarieerde nachtleven (Everett, 1979).

Der nasse Fisch kwam in onze boekhandels onder de titel Schaduw over Berlijn (2018) en de Der stumme Tod als De stille Dood (2019) uit. Het is waarschijnlijk wachten op de nog zes naar het Nederlands te vertalen boeken uit deze GereonRath-Zyklus zoals daar zijn: Goldstein (2010), Die Akte Vaterland (2012), Märzgefallene (2014), Lunapark (2016), Moabit (2017) en Marlow (2018). Der nasse Fisch is ook al omgezet in een graphic novel (Jysch, 2018).

De caleidoscopische kunsttentoonstelling Berlin 1912 - 1932 echter stond stil bij de zich ontwikkelende stedelijke avant-garde die de gruwel van de Eerste Wereldoorlog en de daaropvolgende revolutie op een kunstzinnige wijze trachtte te verwerken. In deze artistieke wervelstorm (Dogramaci, 2018) werden de straat, het geweld, het verraad, de chaos, de politieke polarisatie en de door de economische crisis verpauperde massa met oog voor het sociale en het utopische veruiterlijkt. De tentoonstelling bracht: filmfragmenten, foto's, collages, primitieve videokunst, reclame, architectuur, affiches, pamfletten, tijdschriften, podiumkunst, cartoons, spotprenten en schilderijen. De mythe Berlijn wexd een wereldstad waar nood was aan licht, lucht en zon om de ellende om te zetten in glans die werd gedomineerd door de alom aanwezige genotscultuur (Rossi-Schrimpf, 2018). In de museumboekhandel was naast Kutscher ook de Schotse thrillerauteur Philip Kerr (1956-2018) zichtbaar aanwezig.

Kerr, jurist van opleiding aan de universiteit van Edinburgh, schiep de Berlijnse speurder Bernie Gunther in zijn veertien, best in chronologische volgorde te lezen en naar het Nederlands vertaalde, thrillers. Gunther is: the good cop in the belly of the Nazi beast (Kramer, 2017) die de lezer als opium aan hem bindt. Zijn Berlijn is hell's metropolis (Rankin, 2019). De boeken: Een Berlijnse kwestie (1992), Het handwerk van de beul (1992), Een Duits requiem (1993), De een van de ander (2007), Een stille vlam (2008), Als de doden niet herrijzen (2009), Grijs verleden (2011), Praag fataal (2012), De man zonder adem (2013), De vrouw van Zagreb (2015), De schaduw van de stilte (2016), Pruisisch blauw (2017), Vergeven en vergeten (2018) en Metropolis (2019) hebben steeds oog voor de historische context en overspannen de jaren 1934 tot en met 1957.

Gunther is een sociaaldemocratische inspecteur bij de Kriminalpolizei tijdens de Weimarrepubliek. Door de machtsovername van de nationaalsocialisten wordt hij privédetective, doch hij zal uiteindelijk terug in het nu genazificeerde politie- en veiligheidsapparaat worden opgenomen. $\mathrm{Na}$ de Tweede Wereldoorlog wordt hij opnieuw privédetective en huisbewaarder. In zijn confrontatie met bekende en minder bekende nationaalsocialisten en communisten concentreert zijn al dan niet publieke speurwerk zich vooral op: (serie)moord, ontvoering, afpersing, corruptie, oorlogsmisdaden en georganiseerde misdaad. Gunther is en blijft een tot de verbeelding sprekende cynische en gedesillusioneerde speurder die bovenal tracht te overleven. 
Om te eindigen is een verwijzing naar het werk van de Duitse, voormalige strafpleiter, Ferdinand von Schirach wenselijk. Naast zijn evenzeer in het Nederlands vertaalde thrillers De Zaak Collini (2012) en Taboe (2014) is er de trilogie: Misdaden (2010), Schuld (2011) en Straf (2018). Al deze geanonimiseerde korte verhalen, doorgaans uit zijn eigen praktijk, geven duiding over daders, slachtoffers, criminaliteit, oorzaken en reacties op criminaliteit. Elk verhaal op zich kan studenten criminologie meer dan wegwijs maken in de speurtocht naar goed en kwaad (Grauls, 2019) en het beantwoorden van de schuldvraag (Antonissen, 2019). Ook in zijn Waardigheid is Kwetsbaar (2016) stelt von Schirach op een eerder essayistische wijze pertinente vragen over het functioneren van de rechtsstaat.

Door de interesse in Babylon Berlin en haar in beeld gebrachte Mythos Metropole (Weichenhan, 2018) staat Berlijn weer in de schijnwerpers. Culturele criminologen kunnen in haar politieke, economische, sociale, seksuele en culturele geschiedenis hun hart ophalen om nieuwe inzichten en contextualiseringen in de mogelijke criminologische theaters te (her)ontdekken. Film en literatuur kunnen hierbij helpen.

\section{Bibliografie}

Antonissen, J. (2019), Ferdinand von Schirach. Ex-Strafpleiter en Schrijver met Gezag, Humo, 134-139.

Baqué, E. (2013), A tribute to David Bowie. Hauptstrasse. The Berlin Years 1976-1978. Berlin: Egbert Baqué Contemporary Art.

Boegel, N. (2018), Berlin Hauptstadt des Verbrechens. Die dunkle Seite der Goldenen Zwanziger. München: Deutsche Verlags-Anstalt.

Buchholz, E., R. Hartmann, J. Lekschas \& G. Stiller (1971), Sozialistische Kriminologie. Ihre theoretische und methodologische Grundlegung. Berlin: Staatsverlag der Deutschen Demokratischen Republik.

Cools, M. (2009), 'Das Leben der Anderen' and the Cult of Feliks Dzierzynsky. European Journal of Intelligence Studies, 1, 25-38.

Cools, M. (2018), Curzio Malaparte: een culturele criminologische reis. In: R. De Corte, M. De Vos, P. Humblet, F. Kéfer \& E. Van Hoorde (red.), De Taal is Gans het Recht, Liber Amicorum Willy van Eeckhoutte. Mechelen: Wolters Kluwer Belgium, 459-479.

Diehl, J. (1977), Para-military politics in Weimar Germany. Bloomington: Indiana University Press.

Drogramaci, B. (2018), Nieuwe Berlijners. De metropool Berlijn als artistiek trefpunt in het interbellum. In: I. Rossi-Schrimpf, The New Berlin 1912-1931. Tielt: Lannoo, 43-52.

Everett, S. (1979), Lost Berlin. London: Bison Books.

Feierabend, L. \& T. Kaufmann (2011), Interview Volker Kutscher. Köln: Kölner Stadt-Anzeiger.

Ferrel, J., K. Hayward \& J. Young (2008), Cultural Criminology. London: Sage.

Fijnaut, C. (2014), Criminologie en strafrechtsbedeling. Een historische en transatlantische inleiding. Antwerpen: Intersentia.

Gall, L. (1984), Fragen an die deutsche Geschichte. Bonn: Bundestag.

Gordon, M. (2011), Sündiges Berlin. Die Zwanziger Jahre: Seks, Rausch, Untergan. Wittlich: Index Verlag. 
Grauls, M. (2019), Boekenwijsheid. Vloeibaar goed en kwaad aan elkaar gesmeed. HBvLPLUS, 12 .

Heim, S.; C. Sachse \& M. Walker (2009), The Kaiser Wilhelm Society under National Socialism. Cambridge: Cambridge University Press.

Hirsch, M., D. Majer \& J. Meinck (1997), Recht, Verwaltung und Justiz im Nationalsozialismus. Baden-Baden: Nomos Verlagsgesellschaft.

Jonge, A., de (1980), Kroniek van de Weimarrepubliek. Utrecht: Uitgeverij Het Spectrum.

Jysch, A. (2018), Der nasse Fisch. Hamburg: Carlsen.

Kilb, A., P. Körte (2017), Volker Kutscher im Gespräch. Babylon Berlin - Unsere wilden Jahre. Frankfurter Allgemeine, 12 oktober.

Kramer, J. (2017), The Third Reich's Good Cop. Philip Kerr's Gunther solves crimes for Nazi Germany. Why do we like him so much ?, The New Yorker, 3 juli.

Lekschas, J., H. Harrland, R. Hartmann, G. Lehmann \& G., Stiller (1983), Kriminologie. Theoretische Grundlagen und Analyse. Berlin: Staatsverlag der Deutschen Demokratischen Republik.

Liang, H. (1970), The Berlin Police Force in the Weimar Republic. Berkeley: University of California Press.

MacGregor, N. (2015), Duitsland. Biografie van een natie. Amsterdam: Hollands Diep.

Meador, J. (1986), Impressions of Law in East Germany. Charlotesville: University Press of Virginia.

Mulder, R. (1980), Misdaad en Macht. Criminaliteit, strafrecht en criminologie in de DDR. Amsterdam: Panholzer.

Nippoldt, R. \& B. Pofalla (2018), Night Falls on the Berlin of the Roaring Twenties. Cologne: Taschen.

Ostendorf, H. \& U. Danker (2003), Die NS-Strafjustiz und ihre Nachwirkungen. BadenBaden: Nomos Verlagsgesellschaft.

Rafter, N. (2008), Criminology's darkest hour: biocriminology in Nazi Germany. The Australian and New Zealand Journal of Criminology, 287-306.

Rankin, I. (2019), Introduction. In: P. Kerr, Metropolis. London: Quercus Editions.

Rebbein, K. (1987), Zur Funktion von Strafrecht und Kriminologie im nationalsozialistischen Rechtssystem. Monatsschrift für Kriminologie und Strafrechtsreform, 'Heft 4, 193-210.

Richie, A: (1999), Faust's Metropolis: a History of Berlin. New York: Carrel \& Graf.

Rode, C. (1996), Kriminologie in der DDR. Freiburg: Edition Iuscrim.

Rossi-Schrimpf, I. (2018), The New Berlin 1912-1931. Tielt: Lannoo.

Saft, S. (2008), Justiz und Kriminologie im Nationalsozialismus. Norderstedt: GRIN Verlag.

Salle, G. (2014), Histoire et historiographie de la Kriminologie allemande: une introduction. In: D. Bigo \& L. Bonelli, Critique de la Raison Criminologique. Cultures \& Conflits, L'Harmattan, 241-251.

Siegel, D. (2009), Crime, Music and Politics: Classical Music and Jazz. In: C. Brants \& S. van der Poel, Diverse Kwesties. Liber Amicorum Frank Bovenkerk. Den Haag: Boom Juridische uitgevers.

Ströbele, C. (2017), Die bebende Stadt. Zeit Online, geraadpleegd 17 november 2019.

Stürickow, R. (2019), Kommissar Gennat ermittelt. Berlin: Elsengold.

Töteberg, M. (2018), Von der nasse Fisch zu Babylon Berlin. In: V. Kutscher, Der nasse Fisch. KöIn: KiWi, 547-565.

Watson, P. (2010), The German Genius. Europe's Third Renaissance, the Second Scientific Revolution and the Twentieth Century. London: Simon \& Schuster.

Weichenhan, M. (2018), Berlin-Babylon. Eine deutsche Faszination. Berlin: Verlag Klaus Wagenbach. 
Weitz, E. (2007), Weimar Germany. Promise and Tragedy. Princeton: Princeton University Press.

Wetzel, R. (2000), Inventing the Criminal. A History of German Criminology 1880-1945. Chapel Hill: The University of North Carolina Press.

Wolfe, N. (1992), Policing a Socialist Society. The German Democratic Republic. New York: Greenwood Press.

Wünnenberg, I. (2014), Interview Volker Kutscher. s.l., Verlagsgesellschaft Madsack.

\section{Literatuur}

Doblin, A. (1989), Berlijn Alexanderplatz. Amsterdam: Athenaeum - Polak \& Van Gennep.

Isherwood, C. (1971), Afscheid van Berlijn. Amsterdam: Salamander.

Kerr, P. (1992), Een Berlijnse kwestie. Amsterdam: Meulenhoff Nederland.

Kerr, P. (1992), Het handwerk van de beul. Amsterdam: Meulenhoff.

Kerr, P. (1993), Een Duits requiem. Amsterdam: Meulenhoff.

Kerr, P. (2007), De een van de ander. Amsterdam: De Boekerij.

Kerr, P. (2008), Een stille vlam. Amsterdam: De Boekerij.

Kerr, P. (2009), Als de doden niet herrijzen. Amsterdam: De Boekerij.

Kerr, P. (2011), Grijs verleden. Amsterdam: De Boekerij.

Kerr, P. (2012), Praag fataal. Amsterdam: De Boekerij.

Kerr, P. (2013), De man zonder adem. Amsterdam: Meulenhoff Boekerij.

Kerr, P. (2015), De vrouw van Zagreb. Amsterdam: Meulenhoff Boekerij.

Kerr, P. (2016), De schaduw van de stilte. Amsterdam: Meulenhoff Boekerij.

Kerr, P. (2017), Pruisisch blauw. Amsterdam: Meulenhoff Boekerij.

Kerr, P. (2018), Vergeven en vergeten, Amsterdam: Meulenhoff Boekerij.

Kerr, P. (2019), Metropolis. Amsterdam: Meulenhoff Boekerij.

Kutscher, V. (2018), Schaduw over Berlijn. Amsterdam: The House of Books.

Kutscher, V. (2019), De stille Dood. Amsterdam: The House of Books.

Von Schirach, F. (2010), Misdaden. Amsterdam: Uitgeverij De Arbeiderspers.

Von Schirach, F. (2011), Schuld. Amsterdam: Uitgeverij De Arbeiderspers.

Von Schirach, F. (2012), De Zaak Collini. Utrecht: Uitgeverij De Arbeiderspers.

Von Schirach, F. (2014), Taboe. Utrecht: Uitgeverij De Arbeiderspers.

Von Schirach, F. (2016), Waardigheid is Kwetsbaar. Amsterdam: Uitgeverij De Arbeiderspers.

Von Schirach, F. (2019), Straf. Amsterdam: Uitgeverij De Arbeiderspers. 